\title{
Comparison of the Folstein Mini Mental State Examination (MMSE) to the Montreal Cognitive Assessment (MoCA) as a Cognitive Screening Tool in an Inpatient Rehabilitation Setting
}

\author{
Arun Aggarwal ${ }^{1}$, Emma Kean ${ }^{2}$ \\ ${ }^{1}$ Clinical Associate Professor, Department of Rehabilitation Medicine, Royal Prince Alfred Hospital, Camperdown, NSW, Australia; \\ ${ }^{2}$ Senior Occupational Therapist, Balmain Hospital, Balmain, NSW, Australia \\ Email: arun.a@sydney.edu.au
}

Received August $19^{\text {th }}, 2010$; revised September $14^{\text {th }}, 2010$; accepted September $28^{\text {th }}$, 2010

\begin{abstract}
Identifying the presence of cognitive impairment in patients admitted for inpatient rehabilitation is important as it may impact on their ability to participate in a rehabilitation program. The Folstein Mini Mental State Examination (MMSE) has been used by clinicians as a cognitive screening tool for its convenience, even though it is not sensitive, and often fails to detect cognitive impairment. Recently, the Montreal Cognitive Assessment (MoCA) has been developed as a brief cognitive screening tool to detect mild-moderate cognitive impairment. We performed a longitudinal study to compare the utility of the MMSE and MoCA in an inpatient rehabilitation setting. A total of 50 consecutive patients were studied with an average age of 71.7 years of age. The mean test score for MMSE was 26.5 and 22.2 for the MoCA. The Pearson's correlation coefficient between the scores was $0.695(p<0.003)$. The mean performance time for the MMSE was 7.4 minutes and 14.8 minutes for the MoCA. MMSE did not perform well as a screening instrument for mild cognitive impairment as there were 43 patients who scored 24 or more on MMSE, and 25 patients (58\%) scored 25 or less on MoCA, indicating the presence of cognitive impairment. Even though the MoCA takes longer to administer, the additional important information provided indicates that the MoCA should be used rather than the MMSE as a cognitive screening tool.
\end{abstract}

Keywords: MMSE, MoCA, Rehabilitation

\section{Introduction}

Mild cognitive impairment is common in elderly patients and can impact on prognosis and quality of life [1]. The areas of cognitive impairment that occur at this stage primarily involve attention, verbal fluency, executive function and visuo-spatial skills, which differs from the language and memory skills that are commonly associated with dementia [2]. Identification of mild cognitive impairment in patients admitted for inpatient rehabilitation is important as this may an impact on their ability to participate actively in a multi-disciplinary rehabilitation program and achieve their rehabilitation goals.

Cognitive screening tools in the elderly are important for the purpose of identifying the presence of cognitive impairment. Neuropsychological testing is the gold-stan- dard for assessing dementia and cognitive impairment, but it is time-consuming and requires highly trained assessors [3]. The most commonly used cognitive screening tool, the Folstein Mini Mental State Examination (MMSE) [4], is convenient, but not sensitive, as it is influenced by age, socio-economic status and level of education. It assesses primarily language and memory skills and has been found to be insensitive to detecting mild cognitive impairment [5]. Riedel in a large study of 873 Parkinsons disease patients found the MMSE had a sensitivity of only $50 \%$ when compared to DSM-IV criteria for dementia [6]. Therefore, there is a need for a brief, accurate and easily performed screening test.

The Montreal Cognitive Assessment (MoCA) [7], has been developed as a brief cognitive screening tool to de- 
tect mild-moderate cognitive impairment. It has been found to have high sensitivity and specificity for the detection of mild cognitive impairment. A score of less than 25 was found to be the optimal cut-off point for a diagnosis for mild cognitive impairment.

Hoops and Gill compared the MMSE and MoCA to neuropsychological battery ( $\mathrm{n}=132$ and $\mathrm{n}=38$ respectively), both finding greater ability to detect cognitive impairment using the MoCA. Gill also found good testretest reliability (0.79), inter-rater reliability $(0.81)$ and correlation when compared to a neuropsychological battery $[8,9]$.

This study compares the utility of the MMSE and MoCA in an inpatient rehabilitation setting for everyday clinical use. The aim was to determine the correlation between the MMSE and MoCA scores in individual patients to determine whether the MoCA could be used as a cognitive screening tool to detect mild cognitive impairment.

\section{Method}

Patients transferred to a general sub-acute rehabilitation ward of the Sydney South West Area Health Service between a 6 month period where consecutively recruited into the study. A policy was already in place that patients over the age of 65 had a MMSE performed as part of their conventional rehabilitation assessment. Written consent was obtained to also perform the MoCA assessment on all patients. All assessments were conducted by qualified occupational therapists working on the rehabilitation ward.

Assessment were conducted at least 3 days after being admitted to the ward, so that patients had time to settle into the new ward environment. Patients were examined in an ambient clinical setting. The same therapist performed the MMSE assessment first, followed by the MoCA, on all patients. The time between each test was at least 30 minutes. A modified MMSE using "WORLD” backwards was used for attention testing. Patients who were medically unstable (delirium), had an aphasia, refused or were from a non-English speaking background were excluded from the study. The study was performed with approval obtained though the Human Resources and Ethics Committee of Sydney South West Area Health Service.

Demographic information was collected including ethnicity, language spoken at home, years of education, prior history of dementia, principle diagnosis and diagnosis. The MMSE and MoCA scores were obtained as well as the time taken to perform each test.

All statistical procedures were performed with SPSS 10.0 for Windows. The correlation between MMSE and MoCA results was measured using the Pearson Product Moment Correlation. The Perarson's coefficient reflects

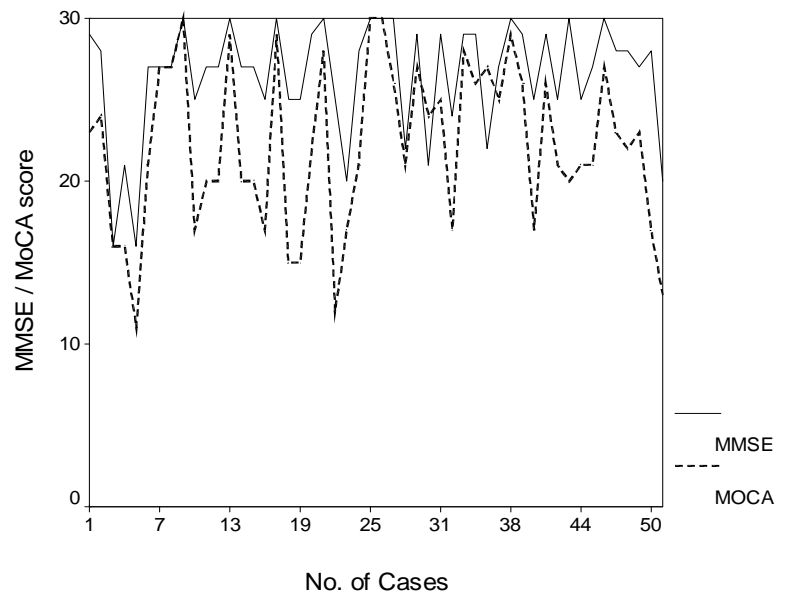

Figure 1. Graph showing comparison of MMSE and MoCA scores.

the degree of linear relationship between the two variables. T-tests were performed on the difference between two dependent samples of results of MMSE and MoCA and time taken to perform these tests.

\section{Results}

A total of 50 patients were studied. The average age of subjects was 71.7 years of age, ranging from 31 to 98 years of age. There were 31 males and 19 females. The mean period of education was 11.7 years with $78 \%$ of subjects completing at least up to Year 10 at school. 67\% of patients completed 12 or more years of education.

The majority primary diagnoses were neurological $42 \%$, (stroke, falls), deconditioned post-operatively 38\% (abdominal and cardiac surgery) and orthopaedic $20 \%$ (total hip and knee replacements). 92\% of subjects spoke English as their primary language at home. The other $4(8 \%)$ spoke another language at home, but had no problems with performing the MMSE and MoCA assessments in English. There was only 1 patient who reported having a previous history of dementia.

MMSE scores ranged from 16 to 30 with a mean MMSE test score of 26.5 with a standard deviation of 3.5. The mean MoCA scores ranged from 11 to 30 with a mean MoCA test score of 22.2 with a standard deviation of 5.1.

The Pearson's correlation coefficient between MMSE and MoCA score was poor at 0.695. A graph showing the comparison between the MMSE and MoCA scores is shown in Figure 1. The difference between MMSE and MoCA scores ranged from 0 to 14 , with a mean difference of 4.6.

The performance time for the MMSE ranged from 4 to 12 minutes with a mean time of 7.4 minutes with a standard deviation of 2.3 minutes. The performance time for MoCA ranged from 5 to 30 minutes with a mean per- 


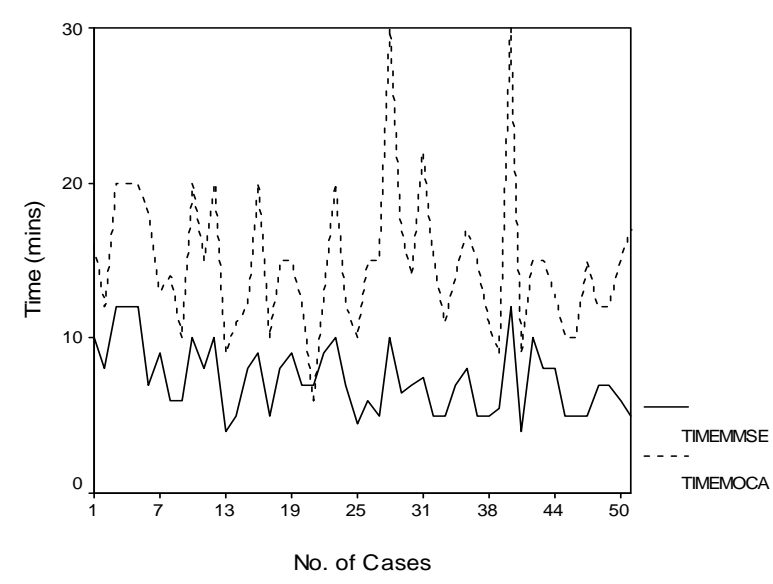

Figure 2. Graph showing comparison between time taken to perform MMSE and MoCA.

formance time of 14.8 minutes with a standard deviation of 4.7 minutes.

The Pearson's correlation coefficient between time taken to perform MMSE and MoCA was also poor at 0.672. A graph showing the comparison between the time taken to perform MMSE and MoCA is shown in Figure 2.

\section{Discussion}

This is a study using MoCA in an inpatient rehabilitation setting and comparing the utility of the MMSE and MoCA in an inpatient rehabilitation setting for everyday clinical use. We found that the MMSE does not perform well as a screening instrument for mild cognitive impairment due in part to the lack of sensitivity to milder cognitive deficits with 10 patients (20\%) achieving a perfect score on MMSE compared to only 3 patients (6\%) on MoCA. In addition, of the 43 patients with intact global cognition, with a "normal" score on the MMSE, defined as greater than or equal to a score of 24,25 patients (58\%) scored 25 or less on MoCA, indicating the presence of cognitive impairment.

Cognitive assessments of inpatients are critical as it influences both long and short term management. Mild cognitive impairment is an intermediate clinical state between normal cognitive aging and dementia, which proceeds and leads to dementia in many cases. The concept of mild cognitive impairment is evolving and somewhat controversial although several screening instruments are available for detecting dementia, the MMSE is the most widely used by clinicians and remains the standard cognitive screening tool in every day clinical practice in many Australian institutions.

However, MMSE is influenced by age, gender, level of education, and socio-economic status. It assesses primar- ily language and memory skills and has been found to be insensitive to mild cognitive impairment. To address this problem, the MoCA was developed as a cognitive tool to screen patients who present with mild cognitive impairment and usually perform in the normal range for MMSE. The data indicates excellent test-retest reliability and positive and negative predictive values for mild cognitive impairment.

Cognitive assessment of inpatients is more challenging than community patients, as intercurrent physical illnesses, deconditioning, increased anxiety, mental illness and noise and distractions, all affect performance. This study however shows that the MoCA is much more sensitive than the MMSE, and even though it is slightly more time consuming, the additional information it provides makes it a more useful clinical cognitive tool that the MMSE.

Even though the MoCA took nearly twice as longer to perform, therapists preferred the MoCA, despite their initial lack of familiarity with the assessment. Clinicians commented the MoCA covers greater areas of cognitive fields and looked at higher executive function, which is not assessed by the MMSE. MoCA was easy to interpret and discuss with other clinicians, compared to more comprehensive cognitive tests such as the Cognistat or a formal neuropsychological assessment. The multiple translations that are available for the MoCA means that it can still be standardized. The score of the MoCA remains out of 30 and therefore makes it a good objective measure for test comparisons and discussion.

Our research suggests that the MMSE does not perform well as a screening instrument for mild cognitive impairment due in part to the lack of sensitivity to milder cognitive deficits with 10 patients (20\%) achieving a perfect score on MMSE compared to 3 patients $(7 \%)$ on MoCA. In addition, of the 43 patients who scored 24 or more on MMSE, 25 patients (58\%) scored 25 or less on MoCA, indicating the presence of cognitive impairment.

Our study recommends that MoCA be used over the widely used MMSE and suggests the need for more validation of the MoCA and MMSE in a population study against other screening instruments. It is a simple stand alone cognitive tool with superior sensitivity to MMSE. It covers important cognitive domains and can be administered generally within 15 minutes and the assessment fits on one page. It is sensitive to the presence of mild cognitive impairment and is feasible for its use in clinical settings. The MoCA promises to fill an urgent and unmet need for brief tool capable of detecting patients with mild cognitive impairment and distinguishing them from the cognitively intact older person. 


\section{Acknowledgements}

We would like to acknowledge the efforts of Mr Martin Oh and Ms Eve Wilson who assisted in performing the cognitive assessments.

\section{Conflicts of Interests}

The authors state that there are no conflicts of interests with regards to this research.

\section{Patient Consent}

Written consent obtained.

Ethics Approval

This study was conducted with the approval of the Human Resources and Ethics Committee Approval of Sydney South West Area Health Service.

\section{REFERENCES}

[1] S. Nazem, "Montreal Cognitive Assessment Performance in Patients with Parkinson's Disease with "Normal” Global Cognition According to Mini-Mental State Examination Score," Journal of the American Geriatrics Society, Vol. 57, No. 2, 2009, pp. 304-308. doi:10.1111/j.1532-5415.2008.02096.X

[2] K. Marder, "Cognitive Impairment and Dementia in Parkinson's Disease,” Movement disorders, Vol. 25, No. S1, 2010, pp. S110-S116. doi:10.1002/mds.22787

[3] C. Zadikoff, S. H. Foz, D. F. Tang-Wai, T. Thomsen, R. M. de Bie, P Wadia, J. Miyasaki, S. Duff-Canning, A. E. Lang and C. Marras, "A comparison of the Mini Mental State Exam to the Montreal Cognitive Assessment in Identifying Cognitive Deficits in Parkinson’s Disease,” Movement disorders, Vol. 23, 2007, pp. 297-299. doi:10.1002/mds.21837

[4] M. F. Folstein, S. E. Foltein and P. R. McHugh, "MiniMental State: A Practical Method for Grading the Cognitive State of Patients for the Clinican,” Journal of Psychiatric Research, Vol. 12, 1975, pp. 196-198. doi:10.1016/0022-3956(75)90026-6

[5] T. N. Tombaugh and N. J. McIntyre, "The Mini-Mental State Examination: A Comprehensive Review,” Journal of the American Geriatrics Society, Vol. 40, No. 9, 1992, pp. 922-935.

[6] O. Riedel, "Cognitive Impairment in 873 Patients with Idiopathic Parkinson's Disease: Results from the German Study on Epidemiology of Parkinson's Disease with Dementia (GEPAD)," Journal of Neurology, Vol. 255, No. 2, 2008, pp. 255-264. doi:10.1007/s00415-008-0720-2

[7] Z. S. Nasreddine, N. A. Phillips, V. Bedirian, S. Charbonneau, V. Whitehead, I. Collin, J. L. Cummings and H. Cherthow, "The Montreal Cognitive Assessment, MoCA: A Brief Screening Tool for Mild Cognitive Impairment," Journal of the American Geriatrics Society, Vol. 53, No. 1-2, 2005, pp. 695-699. doi:10.1111/j.1532-5415.2005.53221.x

[8] D. J. Gill, A. Freshman, J. A. Blender and B. Ravina, "The Montreal Cognitive Assessment as a Screening Tool for Cognitive Impairment in Parkinson's Disease," Movement disorders, Vol. 23, No. 7, 2008, pp. 1043-1046. doi:10.1002/mds.22017

[9] S. Hoops, S. Nazem, A. D. Siderowf, J. E. Duda, S. X. Xie, M. B. Stem and D. Weintraub, "Validity of the MoCA and MMSE in the Detection of Mci and Dementia in Parkinson's Disease,” Neurology, Vol. 73, 2009, pp. 1738-1745. doi:10.1212/WNL.0b013e3181c34b47 